\title{
Linx
}

Revue des linguistes de l'université Paris X Nanterre

$11 \mid 1999$

Typologie des langues, universaux linguistiques

\section{La corrélative et le « quotatif » dans les langues indiennes : deux systèmes qu'on a intérêt à distinguer de la subordination}

Annie Montaut

\section{OpenEdition}

\section{Journals}

Édition électronique

URL : http://journals.openedition.org/linx/883

DOI : 10.4000/linx.883

ISSN : 2118-9692

Éditeur

Presses universitaires de Paris Nanterre

\section{Édition imprimée}

Date de publication : 1 décembre 1999

Pagination : 73-88

ISSN : 0246-8743

\section{Référence électronique}

Annie Montaut, « La corrélative et le « quotatif » dans les langues indiennes : deux systèmes qu'on a intérêt à distinguer de la subordination », Linx [En ligne], 11 | 1999, mis en ligne le 28 juin 2012, consulté le 01 mai 2019. URL : http://journals.openedition.org/linx/883 ; DOI : 10.4000/linx.883

Ce document a été généré automatiquement le 1 mai 2019.

Département de Sciences du langage, Université Paris Ouest 


\title{
La corrélative et le "quotatif» dans les langues indiennes: deux systèmes qu'on a intérêt à distinguer de la subordination ${ }^{1}$
}

\author{
Annie Montaut
}

$1 \quad$ Il est notoire que les descriptions des langues indiennes ont longtemps été obscurcies par l'application de catégories grammaticales empruntées au sanscrit ou au latin ${ }^{2}$, handicap souvent signalé à la comparaison et à la typologie, tant les vernaculaires indiens modernes se sont davantage encore éloignés de l'indo-européen ancien que les langues européennes modernes. C'est en réaction à ce genre de déformation que la typologie moderne aux USA a établi avec Comrie un questionnaire (Lingua 42 1977), visant à l'élaboration de Grammaires Descriptives utilisables pour la comparaison interlangue : le cadre descriptif standard se veut donc explicite, assez général pour couvrir par ses diverses entrées toutes les structures majeures, et assez souple pour rendre compte de la diversité des structures dans les langues. Malgré ses avantages certains ${ }^{3}$, on peut se demander si, dans la présentation des relatives et des subordonnées, il capture parfaitement la spécificité des divers systèmes. Par souci de clarté, je réserverai le terme de subordination aux structures impliquant un subordonnant grammatical sans terme corrélatif dans la principale, désignant simplement par phrase complexe l'ensemble des dispositifs permettant d'intégrer des propositions sémantiquement dépendantes dans une phrase complexe.

\section{Corrélative vs relative, ou corrélation vs subordination}

2 Le problème de la relative, qu'il s'agisse d'ailleurs d'analyses traditionnelles ou modernes, est d'autant plus crucial dans les langues indiennes qu'il est censé permettre de 
départager typologiquement les langues "sans relative» (dravidiennes entre autres) et les langues « à relatives» (indo-aryennes entre autres) ${ }^{4}$. C'est la présence ou l'absence de pronom relatif qui est tenue pour pertinente, plus que la structure de la relative, laquelle est certes commentée mais le plus souvent traitée comme une subordonnée, intégrée, à un rang inférieur, dans la principale, avec ou sans pronom de reprise. En fait, non seulement la 'relative' (du moins déterminative) indo-aryenne relève en réalité d'un système corrélatif, fondamentalement distinct du système de la subordination, mais ce système corrélatif structure l'ensemble du système de la phrase complexe en hindi, langue typiquement indo-aryenne, à l'exception d'un $k i$ «que», vraisemblablement emprunté au persan ${ }^{5}$.

Or on peut hésiter à qualifier de pronom relatif le jo (forme fléchie jis) qui introduit la relative déterminative, car il précède le nom tête --lequel n'est pas antécédent donc--, et s'accorde avec lui comme un adjectif, comme le relatif français « lequel», la proposition entière précédant la "principale » où l'expression relativisée est reprise par le pronom vah (forme fléchie us) "celui-là, il» (1), sauf en cas de focalisation marquée de l'antécédent où l'ordre est inversé (2) :6

1. jo âdmî kal âe un.ko mainne bîs rupâe die 'rel' hommes hier venir-pret-mp ils/ces-dat jeerg vingt roupiesdonner-pret 'j'ai donné 20 roupies aux gens qui sont venus hier'

jo âdmî kal $\hat{\imath} e$ ve paise mâng rahe the

'rel' hommes hier venir-pret-mp ils/ces argent demander progr passé-mp 'les gens qui sont venus hier demandaient de l'argent'

2. ve âdmî paise maang rahe the jo kal âe ces hommes argent demandaient rel hier venir-pret-mp 'ils demandaient de l'argent, les gens qui sont venus hier'

Il s'agit là d'un dyptique corrélatif et non d'une subordonnée et d'une principale, les deux propositions étant certes non indépendantes, co-dépendantes donc, mais sans marque de dépendance hiérarchisée, vah étant corrélé à jo et jo à vah. La protase dans (1) fonctionne un peu comme un thème dont l'apodose constituerait le rhème, le dyptique ayant donc la propriéte de distribuer sur deux propositions la dualité thème / rhème, et par ailleurs le thème y est topique, produisant une information déjà connue ${ }^{7}$. Quant à la relative appositive (3), il est vraisemblable qu'elle est une restructuration du dyptique originaire (1), par inversion (2) puis troncation du pronom de rappel, évolution qu'Haudry (1973) situe à l'origine de la subordination dans les langues romanes, à partir de ce même système de corrélation :

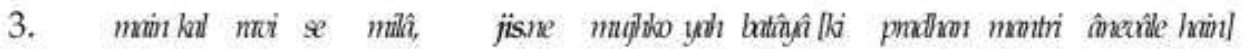

je hier Ravi avec rencontrai, releng jedat ceci adit [que premier ministre va venir] 'hier j'ai rencontré Ravi, qui m'a dit ceci [que le Premier Ministre allait venir]'

De ces deux systèmes, justement soulignés comme radicalement différents par Haudry, le second (subordonnant) s'est imposé, éliminant le premier (corrélation), dans la plupart des langues indo-européennes. Mais en hindi, non seulement le premier demeure bien vivant, mais il continue, comme en sanscrit, à structurer l'ensemble du système de la phrase complexe (comparaison, temps, lieu, hypothèse, etc.), où le premier membre du dyptique est encore introduit par un terme à base $j$ - (issu du sanscrit ya-), et repris dans le 
second membre par un élément à base $s / t$ - (base sanscrite du pronom de troisième personne $)^{8}$, ou $v / u$-, déictique utilisé en hindi moderne pour référer à la troisième personne. Ces paires d'éléments corrélés font aujourd'hui encore paradigme avec l'élément interrogatif à base $k$-, comme en sanscrit, ainsi que le montre la liste suivante, cette base elle-même se trouvant figurer dans le paradigme des indéfinis :

4. jo... vah [kaun ?] qui, jaise... vaise [kaise ?] comme, tel, jitnâ... utnâ [kitnâ ?] autant, jahân... vahân [kahân ?] où, jidhar... udhar [kidhar ?] vers où, jaise hî.. vaise hî (jyõ hî... tyõ hî) dès que, jab...tab [kab ?] quand, jab se... tab se [kab se ?] depuis que, jab tak.. tab tak [kab tak ?] jusqu'à ce que, yadi... to si etc. Indéfinis : koî quelqu'un, kuch quelque chose, kahîn, quelque part, etc.

Quelle que soit la valeur d'origine de ce terme en $y$ - >j-, interrogative ou indéfinie ${ }^{9}$, il est clair, comme le note Haudry, que « c'est la structure binaire qui crée la corrélation, et la corrélation qui crée le relatif », de même que d'autres langues (allemand) montrent que $c^{\prime}$ est la structure anaphorique qui est à l'origine du relatif ${ }^{10}$. Et c'est le système corrélatif qui prévaut encore en hindi moderne, quoiqu'à un moindre degré qu'en sanscrit ${ }^{11}$, système que déforme une analyse qui le décrirait comme subordination (avec conjonction ou complémenteur), et l'isolerait de la corrélative du type (1). Et la morphologie et la syntaxe invitent à comprendre ensemble les séries (5) et (1) :

5a jaise mainne kahâ vaise karo
comme je-erg dis comme fais
'fais comme j'ai dit'

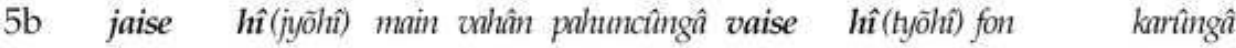
comme dèsque je là j'arriverai comme ès téléphone ferai 'je téléphonerai dès que j'arriverai'

$\begin{array}{lllll}5 c \quad \text { jab } & \text { vah } \text { aegâ } \quad \text { tab batâegâ } \\ & \text { quand il viendra alors dira } \\ \text { 'il (en) parlera quand il viendra' }\end{array}$

5d jab tak râm na $\hat{a} e$ tab tak main yahân rahtungâ quand jusque Ram neg vienne alors jusque je ici resterai 'je resterai ici jusqu'à ce que Ram arrive'

7 Si ce système prévaut, il est concurrencé par la véritable subordination, étrangère à l'indo-aryen et empruntée au persan avec la conjonction $k i$ « que » et ses dérivés, mais il est significatif que ces éléments soient en hindi moderne repris, ou annoncés, par un élément anaphorique, recréant ainsi, à partir d'une structure de subordination stricte, l'ancien système de la corrélative. C'est le cas obligatoirement pour cûnki "puisque », corrélé à islie «c'est pourquoi », agar ${ }^{12}$ « si » (...to « alors»), hâlânki «bien que » (phir bhî " pourtant »). C'est le cas, optionnellement, pour le ki introduisant une complétive après un verbe fini $(6 \mathrm{a}, 7)$, et obligatoire si le verbe introducteur est à une forme non finie (6b) : 


\begin{tabular}{|c|c|c|c|c|}
\hline $\begin{array}{l}\text { usne } \\
\text { il-erg }\end{array}$ & $\begin{array}{l}(y a h) \\
(\text { ceci) }\end{array}$ & $\begin{array}{l}\text { kahâ } \\
\text { dit }\end{array}$ & $\begin{array}{l}k i \\
\text { que }\end{array}$ & $\begin{array}{l}\text { main } \\
\text { je }\end{array}$ \\
\hline
\end{tabular}

7. mujhe aisâ lagâ $k i \ldots$ je-dat ainsi sembla que...

«j'eus l'impression que... "

6b ["sunkar] yah sunkar ki zah kal âegâ mujhe baR̂̀ asharnja huâ (entendant) ceci entendant que il demain viendra je-dat grande surprise fut 'j'ai été très étonné d'entendre qu'il viendait demain'

8 La « complémentation » des noms ("la nouvelle que, le fait que, la décision que", etc.) fait apparaître, obligatoirement, le même déictique, à base $y$-/i-, qui correspond au démonstratif de la proximité, et est systématiquement utilisé pour annoncer ou reprendre un contenu propositionnel :

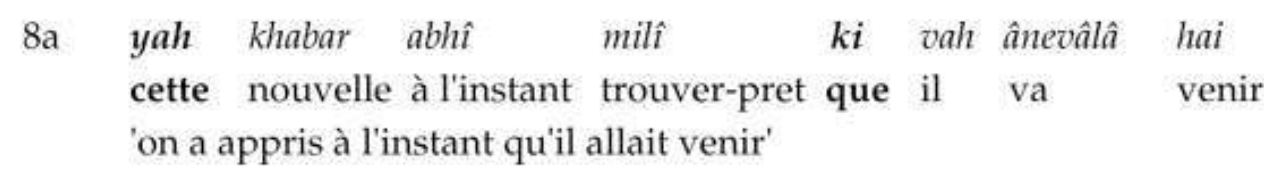

On peut donc estimer qu'il y a eu une corrélativisation généralisée de tout le système de subordination d'emprunt, et s'il est peut-être excessif d'analyser les deux éléments de ces nouveaux dyptiques comme corrélatifs, il l'est tout autant d'analyser le second comme complémenteur ou subordonnant. Le déictique joue un rôle crucial dans le lien entre les deux propositions, comme le montre, en cas d'inversion, la suppression du $k i$ «que», laissant au seul yah « ceci / ce » la charge de lier les deux propositions : ainsi dans ( $8 \mathrm{~b})$ dont les propositions sont inversées par rapport à (8a), et dans (9b) par rapport à (9a) :

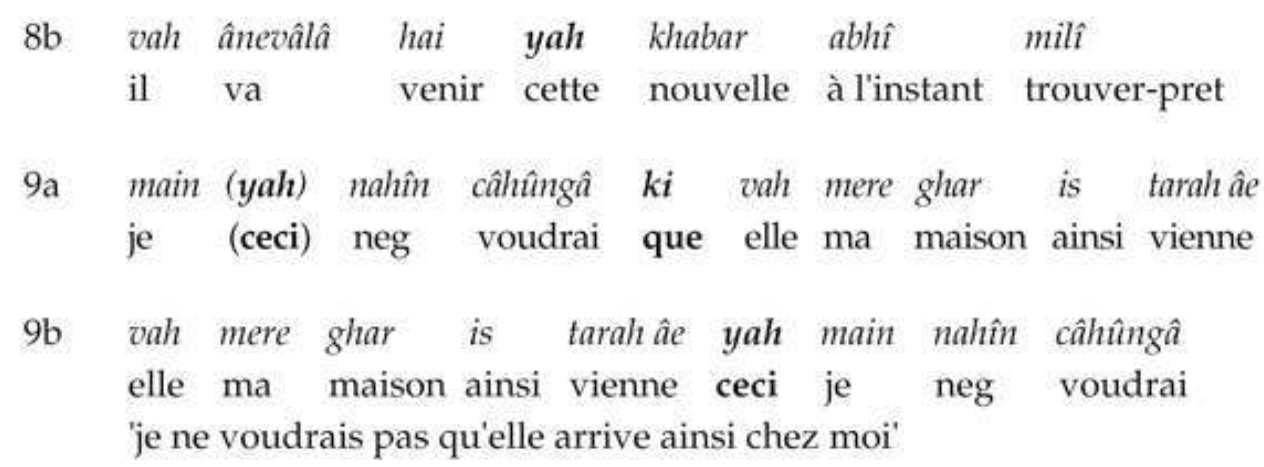

10 Si l'ordre de type (b) est moins fréquent en hindi que l'ordre de type (a), il est par contre très courant en marathi, langue indo-aryenne plus méridionale, où les énoncés (10-11) de Pandharipande (p. 63sq) et (12) montrent le rôle de connecteur du déictique hî, « ce » : 


to gharî âlâ hî bâtmî mîl aiklî
il maison-loc vint cette nouvelle je entendis
'j'ai appris la nouvelle qu'il était revenu chez lui'

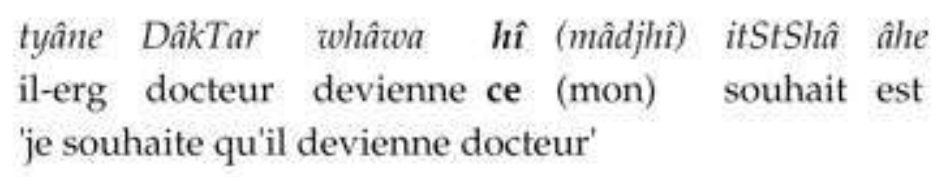

$\begin{array}{llllll}\text { to gharî âlâ hî } & \text { tsangli bâtmî dzhâla } \\ \text { il maison-loc vint ceci } & \text { bonne nouvelle fut }\end{array}$

11 Il est frappant que ce connecteur soit analysé par Pandharipande (1997, p. 63) comme « complémenteur, homophone du déictique »: si une telle analyse peut déconcerter (que faire du ki « que » lorsqu'il est simultanément exprimé dans l'ordre inverse ?), elle traduit un embarras intéressant, révélateur d'une intuition sur le rôle de connecteur qu'assument les anaphoriques en indo-aryen, mais impossible à intégrer dans le format des grammaires descriptives de Routledge. Il ne peut en effet s'expliquer (donc se gloser correctement) que si l'on tient compte de la spécificité du système corrélatif, à l'origine de l'usage des pronoms d'appel ou de rappel employés comme corrélatifs, et, dans le cas de (8-12), de la spécificité des anaphoriques employés comme marque unique d'intégration de la proposition "complétive ", en l'absence du "complémenteur " ki. Faute de tenir compte de ces faits, il est impossible de caractériser correctement les structures de la phrase complexe dans les langues, et de distinguer celles qui organisent la hiérarchie des propositions par la subordination au sens étroit, de celles qui préfèrent la corrélation ou l'anaphore, systèmes qui diffèrent par nature de la subordination ${ }^{13}$.

\section{Le quotatif}

Mais, outre ces deux systèmes, il en est un troisième, tout aussi spécifique, dominant en dravidien mais qu'on rencontre aussi, en concurrence avec la complétive (13b), en marathi (probablement pour des raisons de contact aréal) : c'est une forme figée du verbe " dire », mhaNûn, qui se postpose au contenu de paroles rapporté, lequel précède le verbe principal, jouant en quelque sorte le rôle des guillemets de fin de citation, d'où l'étiquette de « quotatif» :

13a mohan mitrâlâ mi te kâm karin asa/mhaNûn sângat ahe
Mohan ami-dat je ce travail ferai QUOT dit progr. pres
13b mohan mitrâlâ sângat ahe ki mi te kâm karin)
Mohan ami-dat dit progr. pres que je ce travail ferai
Mohan dit à son ami qu'il fera ce travail

Ces exemples, empruntés à Pandharipande ${ }^{14}$, présentent l'adverbe démonstratif asa « ainsi » comme une variante du quotatif, alors que sa catégorie morphologique l'associe plutôt à la série des déictiques connecteurs de (10-12) --la même base en hindi sert aussi d'« annonce » d'une proposition complétive ainsi corrélativisée dans (7)--, et qu'il n'est pas commutable en tout contexte avec mhaNûn. Quand ce dernier a une valeur causale en effet, asa ne lui est pas substituable; du coup l'auteur renonce à la glose quotatif : 
14 to yenar mhaNîn ("asa) mi sagla ghar sadzaula (Pandharipande: 'purpose')

il viendra 'therefore' je toute maison ai-déconé

'I decorated the entire house because he was coming'

Il semble pourtant que mhaNûn soit la même unité fonctionnellement dans (13) et (14), et que asa en diffère dans (13) dans la même mesure que dans (14). Si l'on considère ensemble ce groupe d'énoncés, il apparaît que pour rapporter des propos le marathi a trois possibilités (la complétive en ki, l'anaphore en asa (dispositifs indo-européens), le quotatif en mhaNûn (dispositif dravidien)--le fait que les deux derniers dispositifs aient le même ordre séquentiel explique peut-être l'implantation du système dravidien, mais n'autorise pas à analyser comme tel asa. Le fait même que asa ne soit pas acceptable dans (14) correspond aussi au fait que, en tant que quotatif, mhaNûn ne représente pas une simple cause mais une cause supputée par l'actant, au compte duquel doit être mis le segment de discours correspondant à la cause.

En effet, si l'on se tourne vers le dravidien auquel le marathi a très probablement emprunté le système quotatif, on constate que la valeur causale y est aussi présente, avec de nombreuses autres valeurs circonstancielles. En tamoul, le quotatif (enru, participe conjonctif d'un verbe " dire ») est la seule possibilité d'intégrer un second verbe fini à la phrase, ordinairement à verbe final et unique. On peut donc considérer que l'hypotaxe ne se réalise pas par des moyens syntaxiques ${ }^{15}$ mais discursifs : la valeur considérée comme basique de ce "complémenteur universel " consiste à rapporter des propos ou des contenus de pensée (15), mais les valeurs d'hypothèse, de cause ou d'effet, supposent aussi le passage à une instance d'énonciation distincte :

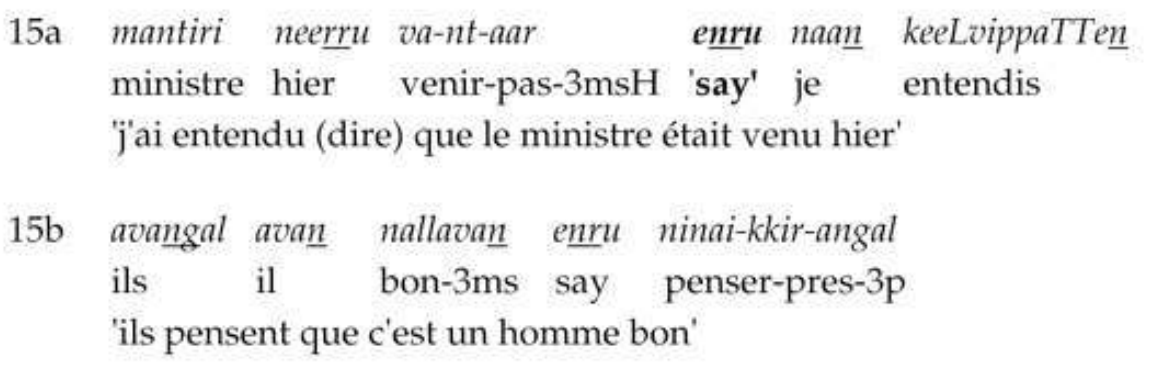

16. kumaar nallavaan enraal avanaik kuuppiTvoom

Kumar bon-3ms say-cond il-acc appeler-fut-1p

'si Kumar est bien nous l'inviterons' （Lehmann 1989)

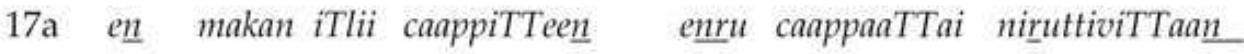
mon fils idli manger-pas-1s say repas a laissé / sauté 'mon fils a sauté le repas parce qu'il avait mangé des idli' (Rama Rao 1972)

16 L'hypothèse de (16) reflète l'avis du protagoniste ou l'avis général, la cause de (17a) est celle que l'énonciateur principal attribue à l'actant "mon fils», ainsi que le montre l'emploi de la première personne du verbe «manger». La version interrogative de ce dernier énoncé $(17 b)$ fait du reste apparaître une préférence pour la première personne (discours direct de celui à qui est attribuée la relation causale, l'enfant, distinct de l'énonciateur principal, la mère), signe selon Rama Rao (1972, p. 146) que enru causal «marque quelque chose de plus que la simple cause ». De même (18) emprunté à Larkin 
(1972, p. 43) ne marque pas une cause factuelle objective mais le prétexte sous lequel la police a arrêté le supposé voleur (« on the basis that ») :

\section{7b iTlii caappiTTeen (? caappiTTaay) enru caаppaaTTai nï̈uttiviTTaayaa? idli manger-pas-1s (? manger-pas-2s) say repas sauter-pas-2s-interr 'as-tu sauté ton repas parce que tu as mangé des idli ?'}

\section{8. paNattai tiruTinaan $\underline{\text { enru }}$ pooliicaar avanaik kaituceytaarkaL argent-acc voler-pas-3ms say policiers il-acc arrestation ont fait 'la police l'a arrêté parce [alléguant] qu'il avait volé l'argent'}

Bref, comme le note nettement Larkin, mais dans une perspective strictement centrée sur l'établissement du statut syntaxique de l'objet de enru, enru " enchâsse des segments de parole » (piece of language), et ces "enchâssements sont attribués à quelqu'un », sous forme de "pensée, de description de sentiment ou d'état d'esprit »(p.44). Disons qu'enru implique l'introduction d'un segment discursif produit par un sujet de conscience distinct de l'énonciateur principal, ce qui explique en particulier qu'il se distingue de la cause présentée comme factuelle: une relation "causale » dominée par enru peut très bien admettre une contrepartie factuelle sans contradiction, alors qu'une relation causale objective (verbe non fini et suffixe causal) ne le peut pas :

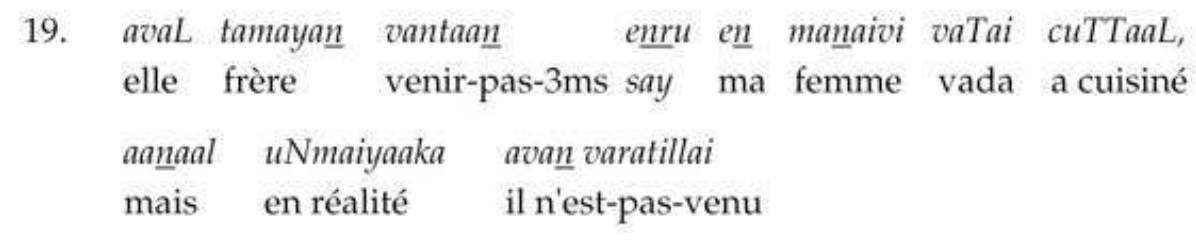

'ma femme a préparé des vada parce [croyant] que son frère venait, mais en fait il n'est pas venu' (ou "a allégué la venue de son frère pour justifier la préparation des vada ») ex de Rama Rao 1972: 146-7

Par conséquent, même quand il n'est pas en emploi quotatif stricto sensu - rapportant des paroles ou des pensées -, enru garde une valeur logophorique, du reste inscrite dans la forme. En tant que dispositif d'insertion de propositions finies à diverses valeurs circonstancielles, il conserve la propriété, inconnue du système corrélatif ou subordonnant de l'indo-aryen ${ }^{16}$, d'introduire une deixis centrée sur l'ici/maintenant d'un autre énonciateur. Ceci implique deux choses : 1) en tant que "complémenteur » enru reste un dispositif de changement d'instance dénonciation, et il n'y a pas de raison de le gloser et de l'analyser différemment que le quotatif de (15) ; 2) l'introduction d'un second verbe fini en dravidien est strictement conditionnée par le recours à une seconde instance d'énonciation ${ }^{17}$, comme si, pour hiérarchiser les niveaux logiques de la phrase complexe, il fallait passer par une réattribution de la parole, et introduire un point de vue énonciatif autre, l'énoncé simple correspondant à une instance d'énonciation et réciproquement. Une proposition = une énonciation. La phrase complexe étant toujours un emboîtement de plusieurs niveaux d'énonciation, on peut dire que la hiérarchie des propositions, qui est encodée dans la syntaxe dans certaines langues, l'est dans le discours en dravidien, au sens que Benveniste a donné au terme (sphère déictique de l'énonciateur, ce qui relève de son ici-maintenant). Hiérarchiser les propositions correspond donc à changer de référence déictique. C'est là une différence typologique majeure entre langues à subordonnants et langues à quotatif ${ }^{18}$, dissimulée par l'habillage en « complémenteur » ou « conjonction » du quotatif. 

$\mathrm{d}^{\prime}$ « enchâsseurs de prédicats finis $»^{19}$ en dravidien, partagent significativement ces propriétés. Significativement aussi, enru peut complémenter certains types de noms ('rumeur', 'loi', 'nouvelle', 'souci', 'ordre'), de type logophorique, et non d'autres, de type évenementiel ('résultat', 'occasion', 'événement'). Dans ce cas, la forme, aussi figée, de participe passé enra, peut commuter, par exemple comme insertion du complément de «rumeur » (vadanti), présentant alors le fait comme un déjà dit, le rappel d'une information déjà connue, alors que dans le même contexte enru présente une information nouvelle : Larkin (1972) oppose ainsi « il s'éleva une rumeur que (enru / * enra) le ministre s'était suicidé » à «la rumeur que (enra / *enru) le ministre s'était suicidé s'évanouit ». Kothanandaram (1981) développe ces exemples et d'autres analogues qui confirment ce type d'opposition, mais dans une perspective strictement syntaxique (de quoi doit-on dériver enra ?). Ces distinctions finalement aspectuelles correspondent à la présence implicite d'un énonciateur qui n'a pas le même point de vue sur l'énoncé qu'il rapporte, énoncé par ailleurs rapporté lui-même dans le mêmes termes ${ }^{20}$.

Certes, on peut noter une certaine grammaticalisation du quotatif, dans le flottement des marques déictiques. Larkin, toujours dans le même article (p.61), cite un contraste révélateur, à propos du verbe "critiquer (le fait) que », "désapprouver que », qui peut prendre comme complément un verbe non fini à l'accusatif et aura une interprétation «factuelle» (dans (20a) c'est <mon comportement> qui est critiqué), ou un segment de discours à l'impératif introduit par enru, et il aura alors une interprétation logophorique : dans (20b) c'est la critique qui est rapportée au style direct, à l'impératif. Mais il peut aussi comporter un complément inséré par enru sans changement de sphère déictique dans (20c), l'interprétation étant non logophorique (le fait que je fasse), plus proche donc de (20a) que de (20b) :
naan elunDu
veLai cey.tat.ai
DaakTar kaNTittaar
1s se-lever-part.conj travail faire.nom.acc docteur critiquer-pas-3sH

'le medecin a critiqué le fait que je me lève et aille travailler' (Larkin : 'my getting up and working')

20b nii elunDu veLai ceyyakkuuTaaTu enru DaakTar kaNTitaar 2s se-levant travail faire-imper-neg say Docteur a critiqué 'le médecin a désapprouvé que je me lève et aille travailler (" criticized saying that I should not... " dit Larkin, ou même « disant, toi, ne vas pas travailler " : m'a déconseillé d'aller travailler)

\section{0c naan eluNDu veLai ceiteen enru DaakTar kaNTitaar 1s se-levant travail faire-pas-1s disant Docteur a critiqué "le docteur a désapprouvé le fait que je me lève et aille travailler "}

Semblable grammaticalisation, qui peut justifier une réanalyse du quotatif comme complémenteur, se retrouve dans les langues indo-aryennes qui ont emprunté le système quotatif, et s'y explique par ailleurs par la concurrence du système indigène de subordination. Cependant, vu l'importance (encore aujourd'hui) ${ }^{21}$ des marques formelles de réattribution de la parole dans les segments insérés par enru, vu aussi qu'on ne trouve les « quotative referents » que dans les phrases enchâssées par en- (Larkin 1972, p. 45), il semble souhaitable de lui conserver sa spécificité dans la glose et dans l'analyse ${ }^{22}$, seule 
manière de mettre en évidence les distinctions typologiques signalées ci-dessus, entre hypotaxe par dispositif syntaxique et discursif.

\section{Conclusion : les conditions d'un calque}

On peut pour finir se demander comment et pourquoi des langues qui ont un dispositif syntaxique bien développé pour hiérarchiser les propositions complexes ont été amenées à emprunter un dispositif radicalement distinct, fondé qu'il est sur la réattribution de la parole (dédoublement des instances d'énonciation) comme unique moyen de faire des phrases complexes à verbes finis. Parmi les explications qu'on peut donner du fait, le contact a certes un rôle important, mais n'explique pas à lui seul un emprunt si extraordinaire ${ }^{23}$.

Si l'on revient sur les données de la première partie, la série (13) du marathi montre une identité positionnelle entre le quotatif mhaNûn et l'anaphorique asa. Si le premier est structurellement typiquement dravidien, le second est typiquement indo-aryen, s'inscrivant dans le dispositif de connexion par anaphore, dont on a vu les rapports qu'il entretient avec le système de corrélation, typiquement indo-aryen. Corrélation et anaphore peuvent en effet être vues comme une aryanisation de la subordination en $k i$ «que », comme l'indiquent les exemples (10-12) du marathi et (8-9) du hindi. Le système en hindi n'est que rarement de la forme ( $9 b)$ c-à-d. proposition "dépendante » anaphorisée par le déictique et directement suivie de la $"$ principale $»^{24}$, présentant plus souvent l'ordre (9a) «principale » et élément cataphorique du ki «subordonnant». En marathi par contre, qu'on parle ou non d'inversion du dyptique et de troncation (du «subordonnant» ki), il est bien plus fréquemment du premier type, c'est-à-dire que l'élément anaphorique se trouve directement postposé au contenu propositionnel "dépendant ", comme dans (10-13a). L'anaphorique est régulièrement dans la position qu'occupe enru en dravidien, facilitant la prise du calque du quotatif (13a). Cet ordre est du reste en conformité avec l'ordre de base attendu d'une langue à « tête finale », le verbe principal dominant les autres prédicats ${ }^{25}$. En outre, comme le constituant interrogatif dans l'interrogation totale domine lui-même toute la phrase, on peut s'attendre à le trouver en position finale, et c'est le cas en dravidien, comme en marathi, mais non en hindi, qui n'a justement pas intégré la structure du quotatif :
21a kumaar raajaa-v-ai aTi-tt-aan-aa
" est-ce que Kumar a tué le roi ? "
$21 \mathrm{~b}$ to $\mathrm{kal}$ parat aalaa kay
il hier re-est venu interr
« est-ce qu'il est revenu hier?"
21c kyaa kumaar raajaa ko maaraa
interr Kumar roi acc tua
" est-ce que Kumar a tué le roi ?"

kumar roi-acc frapper-passé-3ms-interr

24 Deux conditions semblent donc expliquer l'intégration du système quotatif en indoaryen: la présence du système corrélatif dans sa version anaphorique, et l'ordre des 
propositions, la protase devant être celle qui est anaphorisée dans l'apodose et jouer le rôle logique de complétive, ordre conforme au type rigide à tête finale ${ }^{26}$.

Il est clair que la mise en évidence de ces fonctionnements et de leur histoire suppose une description correcte de chacun des dispositifs en jeu, et la description correcte suppose une identification des spécificités de chacun, souvent liées à l'histoire de la forme dans sa morphologie même, et toujours à l'ensemble de ses occurrences dans l'économie générale de la langue. C'est ce à quoi ne permet pas d'aboutir une grille d'analyse où la relative apparait disjointe des subordonnées dites adverbiales (circonstancielles), et où le quotatif ne se trouve raccolé que par la tangente des complétives.

\section{BIBLIOGRAPHIE}

AgESTHIALINGOM, S. (1967) A General Grammar of Tamil, Annamalai Nagar, Anamalai Nagar Press

BAZIN, L. (1978) Introduction à l'étude pratique de la langue turque, Paris, A. Maisonneuve

BLOCH, J. (1924) « Sanscrit et dravidien », BSLP, 25 pp.1-21

BLOCH, J.(1970) The Formation of Marathi Language, Delhi, Motilal Banarsidass [1919, ed. fr]

Emeneau, M.B. (1980) Essays on language and linguistic area, Stanford, Stanford Univ. Pr.

GondA, J. (1954) « The Original Character of the IE Relative Pronoun », Lingua, 4 (pp. 1-41)

GREENBERG, J.H. (1966) « Some Universal of Grammar with particular reference to the order of meaningful elements », in Greenberg J.H. (ed.) Unversals of Language, Cambridge, MIT Press (pp. 73-113).

GuPTA SAGARMAL, (1986) Discourse Grammar of Hindi : a Study on relative Clauses, New-Delhi, Bahri Publ., SIL 15

HAUDRY, J. (1973) « Parataxe, hypotaxe et corrélation dans la phrase latine », BSLP LVXVIII, pp. $147-86$

KHAIDER, M. (1980) Dakkhini, Mysore, Central Institute of Indian Languages Publ.

Kothanandaram, P. (1981) « The Syntax of enru and enra in Tamil », in Agesthialingom S. \& N. Rajasekharan Nair (eds.) Dravidian Syntax, Annamalai Nagar, Annamalai (pp. 77).

LALAS S. (1997) Râjâsthânî Vyâkaran [Grammaire du Rajasthani], Jodhpur, Rajasthani Shod Sansthân Caupâsanî

LARKIN, D. (1972) Enru and enpatu as complement markers in Tamil, in Agesthialingom S. \& S.V.

Shanmugaan (eds.),Third Seminar on Dravidiaan Linguistics , Annamalai Nagar, Annamalai Univ.Pr. (pp. 37-74)

LeHmanN, T. (1989) A Grammar of modern Tamil, Pondicherry Institute of Linguistics and Culture, Pondicherry

MinARD, A. (1936) La Subordination dans la prose védique, Paris, Belles Lettres

MontAut, A. (ed.) (1997), Les Langues d'Asie du sud, Faits de langues 10, Ophrys 
Montaut, A. (à paraître) « Ordre des constituants et hypotaxe dans les langues indiennes », Vème journée d'Etudes en linguistique de l'Inalco (l'ordre des termes), Cahiers du CLI

PANDHARIPANDE, R. (1997) Marathi. A Reference Grammar, Londres, Routledge

RAmA RAO, C. (1972) « Causal Use of Quotative Morpheme in Dravidian », in Agesthialingom S. \&

S.V. Shanmugaan (eds.),Third Seminar on Dravidian Linguistics , Annamalai Nagar, Annamalai

Univ.Pr. (pp. 37-74)

RAMASAMY, K. (1981) Correlative relative clauses in Tamil, in Agesthialingom S. \& N. RAJASEKHARAN N

AIR (eds.) Dravidian Syntax, Annamalai Nagar, Annamalai (pp. 363-80)

ReNOU, L. (1930) Grammaire du Sanscrit, Paris, Maisonneuve

Southworth, F. (1971) « Detecting prior Creolization : An Analysis of the Historical Origins of Marathi », in Pidginisation and Creolization of Languages, D. Hymes (ed.), London, Cambridge Univ. Pr.

SteEVer, S. (1991) «Exceptions to Case Marking and the Grammar of Complementation in Dravidian ", Studies in Dravidian and General Linguistics, A Festshrift for Bh. Krishnamurti, Hyderabad, Center of Advanced Study in Linguistics, Osmania University Publications in Linguistics (pp. 198-214)

\section{NOTES}

1. Je remercie Georges Rebuschi pour les commentaires qu'il a apportés à la première version écrite de ce texte.

2. Dans les grammaires rédigées par les grammairiens traditionnels indiens ou les missionnaires. Ainsi la structure ergative y a souvent été analysée comme passive, les structures expérientielles à sujet au datif n'ont pas reçu l'attention qu'elles méritaient, moins encore les divers énoncés possessifs, alors que la «flexion nominale» (deux formes en hindi, et pour certains paradigmes seulement, les autres ne variant pas) restait décrite avec huit cas comme en sanscrit.

3. En l'occurrence pour les langues indiennes, l'identification de la structure ergative, distincte du passif comme de l'actif, des prédications dites à « datif subject », etc.

4. De la famille dravidienne, parlée au sud de l'Inde, le tamoul est pris ici comme exemple, de la famille indo-aryenne, parlée dans le nord et le centre et issue du sanscrit, sous groupe de l'indoeuropéen, le hindi. Les langues des deux familles (comme celles du groupe tibéto-birman et austro-asiatique) partagent aujourd'hui de nombreux traits typologiquement importants, comme l'ordre dominant des constituants (SOV) - alors que l'ordre des termes n'était pas contraint en sanscrit - et la présence de consonnes rétroflexes, notées ici par la majuscule (voir Montaut 1997, «problématique d'ensemble »).

5. Et de ses dérivés : tâki « afin que », kyonki « parce que », jab ki « alors que, hâlânki « bien que », cûnki « puisque ».

6. L'emploi des guillemets («principale») indique que le terme n'est pas strictement approprié, mais qu'un autre (comme ici apodose) ne le serait guère plus (voir ex 2). Sur la relative en hindi, voir Gupta 1986. Principales abréviations : pres (présent), pret (préterit), pas (passé), progr (progressif), erg (ergatif), dat (datif), acc (accusatif), loc (locatif), rel (relatif), part.conj. (participe conjonctif), $\mathrm{m}, \mathrm{f}, \mathrm{n}, \mathrm{s}, \mathrm{p}$ (genre et nombre), $\mathrm{H}$ (honorifique).

7. Gupta (1986) souligne justement le caractère topique du premier élément du dyptique. Que le co-énonciateur partage ou non réellement l'information, elle lui est présentée comme telle. La structure thème-rhème assure par ailleurs la cohésion interne du dyptique soulignée par les morphèmes corrélatifs. 
8. Cette ancienne base ne survit plus aujourd'hui que dans des éléments adverbiaux (so « ainsi », tab «alors ») ou dans des formules archaïques comme pronom de reprise : jo ho gayâ so ho gayâ (rel a été cela a été) 'ce qui devait arriver est arrivé, ce qui s'est passé est passé'.

9. Sur laquelle je reviens plus en détail dans une étude sur «l'ordre des constituants et l'hypotaxe dans les langues indiennes » (à paraître).

10. On trouve d'ailleurs en rajasthani, autre langue indo-aryenne occidentale, un système d'anaphoriques comme variante de la corrélative (le pronom relatif jika, jiko, jai, alternant avec le déictique de la proximité aa, corrélé à l'autre démonstratif vi/vo : Lalas 1997).

11. Où « la subordination n'existe guère... », car « le trait significatif des subordonnées est d'être des corrélatives », indique Renou (1930, § 391 sq.), comme Minard (1936), consacrant la première partie de son ouvrage au dyptique, la seconde (les subordonnants) menant en parallèle l'étude du «subordonnant » comme yavat et du corrélatif comme tavat.

12. agar est emprunté au persan mais conserve la structure (corrélative) du yadi, « si », indigène.

13. Et dont il est peut-être sûr qu'ils la précèdent et en constituent l'origine (latin, français et langues europénnes), mais non que cette évolution soit linéaire, comme le suggère Haudry : la reconquête corrélative par la corrélativisation des complétives en est un bon exemple en hindi moderne.

14. P. 5 sq. Le sujet et l'objet (mohan mitrâlâ) peuvent directement précéder le verbe principal : mi te kâm karin asa/mhaN ${ }^{1} n$ mohan mitrâlâ sangat ahe.

15. Ni même strictement morphologiques : Steever 1991 voit dans ce dispositif de FPE, «finite predicate embedders ", la seule exception au principe que tout terme doit recevoir un cas assigné par le verbe unique, les verbes dépendants étant donc sous une forme nominale, non finis :

mantiri neerru va-nt-at-ai naan keeT-T-een

[ministre hier venir-pas-nom-acc 1s entendis dire]

'j'ai entendu (dire) que le ministre était venu hier'

$17 \mathrm{~b}$ kumaar vantaal naan avanitan peeruveen

Kumar venir-part.cond je il-loc parler-fut-ls (verbe venir non fini)

'si Kumar vient, je lui parlerai'

16. Qui n'exhibe cette propriété que lorsqu'il s'agit de citation au sens strict de paroles ou de pensées. voir (6).

17. De façon très marginale, certains suffixes dits focalisateur $(-e e)$ ou topicalisateur (-oo) permettent aussi d'enchâsser un prédicat fini. Voir note $25 \mathrm{c}$.

18. Dont le quotatif est utilisé comme subordonnant universel.

19. La catégorie des FPE de Steever (1991) : pol, « sembler », et agu « devenir ».

20. Les emplois littéraires de enru introduisant des comparaisons «illogiques » (Rama Rao dans une discussion du trait +/- animé du sujet principal) vont aussi dans ce sens : dire « il a comme plu des fleurs parce que tu es venue » signifie qu'un énonciateur fait le lien entre la métaphore et sa cause, et non qu'il y a un rapport causal objectif :

nii ciikiramaka vantany enru puu malai polintatu
2s tôt venir-pas-2s say fleur pluie a paru
'il a plu desfleurs parce que tu es venue tôt'

21. Il y aurait évidemment une étude diachronique à faire sur ce point.

22. Les emplois de enru quand il n'introduit pas un verbe fini, qui mériteraient à eux seuls une analyse poussée, ne peuvent se comprendre ensemble que si on tient compte de sa fonction de «shifter " énonciatif si l'on peut dire. 1) après un segment onomatopéique, 2) «emphatique " après un terme au datif auquel il donne le sens de "justement, particulièrement pour », 3) «adverbial» au sens «en tant que, comme»(donner 10 roupies comme commission), 4) «intensif» quand il introduit un radical verbal redoublé (frapper <frappe frappe enru> = «frapper violemment », 5) «et ainsi de suite " après une énumération. 1) isole un segment hétérogène au code et signale cette hétérogénéité comme s'il s'agissait d'une énonciation distincte, 2) fait du segment au datif une unité que l'énonciateur choisit de souligner comme 
unique (pour $\mathrm{X}$, je dis bien pour X), d'où l'effet de focalisation restrictive, 3) fait état d'une équation (as, as if) forcément mise au compte de l'actant qui considère les 10 roupies comme commision, 4) correspond à peu près à «ce qu'on appelle battre", 5) suppose un retour sur le message pour rendre la liste ouverte (aadi en hindi et sanscrit, "premier, initial » revient aussi sur les mots précédent pour les présenter comme le début d'une liste).

23. Les contacts entre dravidien et indo-aryen ont fait l'objet de solides études (de Bloch 1924 à Emeneau 1980), l'histoire du marathi en fournissant un exemple particulièrement net (Bloch 1919).

24. Les guillemets marquent que toutes deux sont non indépendantes et non du type subordonnée-principale bien qu'il soit délicat de les assimiler complètement aux correlatives de (1-5).

25. Voir pour plus de détails « ordre des constituants et hypotaxe dans les langues indiennes », Vème journée d'Etudes en linguistique de l'Inalco (l'ordre des termes), à paraître.

26. a) On peut noter qu'en hindi méridional, parlé en milieu dravidophone (qui a intégré le système quotatif), les pronoms de rappel qui anaphorisent en hindi standard la relative, en sont venus à remplacer la structure corrélative, se postposant au verbe sur le modèle des participes dits relatifs du dravidien : aya so admi, [venu-démonstratif homme], «l'homme qui est venu », au lieu de jo admi aya voh... [lequel homme est venu il...] (Khaider 1980). L'anaphorique, lié au système corrélatif, semble bien un relais pour les calques du dravidien impliquant strictement l'ordre à tête finale (voir note 9).

b) Il est aussi une structure en hindi standard oral qui paraît en voie de réanalyse analogue : l'hypothétique "si » en standard écrit est toujours repris par un «alors » to, à l'initiale de l'apodose, et fréquemment omis. Si bien que la norme devient pour exprimer l'hypothèse : hypothèse to apodose, et en cas de phrase suspensive (" (et) s'il n'était pas venu..../ ? ",vah na aayaa hotaa to [il ne serait pas venu to ], ce dernier élément étant intonativement intégré à la proposition qui précède.

c) Symétriquement le calque de la corrélative en tamoul (rare, l'interrogatif tenant lieu de relatif), passe par un dispositif emphatique (clitique focalisant -ee).

\section{AUTEUR}

\section{ANNIE MONTAUT}

Inalco 